\title{
Outcomes and Complications of Double Roux Loop Reconstruction in Pancreaticoduodenectomy: A Single Center Experience
}

\author{
Abolfazl Afsharfard ${ }^{1}$, Barmak Gholizadeh (iD ${ }^{2,{ }^{* *}}$, Behzad Azimi ${ }^{2}$, Mohammad Amin Shahrbaf ${ }^{2}$ and \\ Adel Zeinalpour (iD) ${ }^{2, *}$ \\ ${ }^{1}$ Department of General and Vascular Surgery, Shahid Beheshti University of Medical Sciences, Tehran, Iran \\ ${ }^{2}$ Department of General Surgery, Shahid Beheshti University of Medical Sciences, Tehran, Iran \\ "Corresponding author: Department of General Surgery, Shahid Beheshti University of Medical Sciences, Tehran, Iran. Email: adel.zeinalpour@gmail.com \\ "* Corresponding author: Department of General Surgery, Shahid Beheshti University of Medical Sciences, Tehran, Iran. Email: barmak.gholizadeh@gmail.com \\ Received 2019 March 09; Revised 2019 May 23; Accepted 2019 May 26.
}

\begin{abstract}
Background: pancreaticoduodenectomy is associated with higher morbidity and mortality rates. The leak of activated pancreatic enzymes can cause several complications which could be life threatening. One of the suggested methods, which can prevent complications, is double Roux anastomosis technique.

Objectives: The aim of this study was to evaluate the outcomes and complications of double Roux anastomosis method.

Methods: In this prospective study, patients who underwent pancreaticoduodenectomy with double Roux anastomosis were evaluated from 2013 to 2017. The outcomes and complications such as mortality rate, pancreatic leak or fistula, bile leakage, and abscess formation were evaluated.

Results: A total of 12 patients were evaluated. The mean age was $53.08 \pm 13.43$ (19-70). Seven of them were male and five were female. Indications of surgery in the patients were periampullary cancer ( 6 patients), pancreatic head cancer (4 patients), distal cholangiocarcinoma (1 patient) and duodenal trauma (1 patient). There was no mortality, no pancreatic leak or fistula, no hemorrhage, and no abscess in any of the patient. There was one case of the biliary leakage, which was managed expectantly.

Conclusions: The results of this study showed that use of separate double Roux, one for the pancreas and the other one for the stomach and bile duct reduces complications and mortality. Although this method requires more anastomosis and the operating time is prolonged, but ultimate outcome of the patient has great effects.
\end{abstract}

Keywords: Pancreaticoduodenectomy, Anastomosis, Roux-en-Y

\section{Background}

Whipple procedure or pancreaticoduodenectomy is a surgical procedure for patients with neoplasms of the pancreatic head, ampulla of Vater, and distal parts of the common bile duct. This procedure was first introduced in 1912 (1-3). During the surgery, after pancreaticoduodenectomy, gastrointestinal tract reconstruction is carried out by gastric, biliary, and pancreatic anastomosis to the jejunum (4). Whipple procedure has been associated with a mortality rate of $<5 \%$ and a morbidity rate of $25 \%$ to $60 \%$ (5-8).

The most common complication of following Whipple procedure is pancreatic fistula with the incidence of $5 \%$ to $30 \%$ in postoperative period $(9,10)$. Pancreatic fistula causes complications such as intra-abdominal abscess, sepsis, and life-threatening intra-abdominal hemorrhage (10-12). The most important pathophysiology for the complications of Whipple procedure is activation of pancreatic secretions by bile in the Jejunal limb. So that, in the anastomosis leakage, activated pancreatic enzymes could cause many damages to intraperitoneal and retroperitoneal tissues (13).

Double Roux reconstruction procedure (DRRP) method has several advantages such as decrease in the stasis of pancreatic secretions, prevention of the pancreatic enzymes activation by bile, and enterokinase and acidotic gastric secretions. In addition, anastomotic leakage in DRRP would be milder and easy to manage than conventional Whipple procedure $(14,15)$.

\section{Objectives}

The aim of the current study was to evaluate outcomes and complications of pancreaticoduodenectomy with DRRP. 


\section{Methods}

\subsection{Study Design}

This prospective study was done on patients who underwent pancreaticoduodenectomy with DRRP in Shohadaye Tajrish Medical Center, Shahid Beheshti University of Medical Sciences, Tehran, Iran from May 2016 to May 2017. The study population included patients who referred to Shohadaye Tajrish Medical Center for pancreaticoduodenectomy. The patients provided written informed consent to undergo DRRP and the study protocol was approved by the institutional review board. Furthermore, a checklist was used to collect the information, which included age, gender, complications, and mortality of the patients.

\subsection{Surgical Technique}

During the conventional Whipple procedure, in the first phase, distal part of the common bile duct(CBD), head of pancreas, duodenum, and jejunum up to $10 \mathrm{~cm}$ distal to the ligament of treitz were resected. In the reconstruction phase, a single limb of jejunum was used for reconstruction (Figure 1) so that all 3 anastomoses were done on this limb of jejunum.

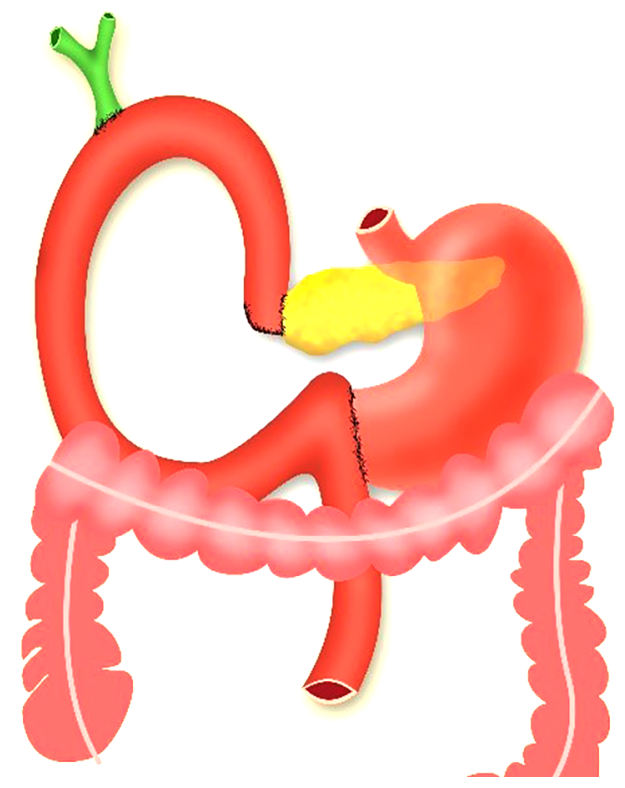

Figure 1. Single loop reconstruction in Whipple surgery

However, during DRRP and after resection, in the reconstruction phase, the double Roux limb (Figure 2) was used for reconstruction instead of a single limb.

In this study we drawn up the first Roux arm from retro-colic space to anastomosis with the pancreas. For end

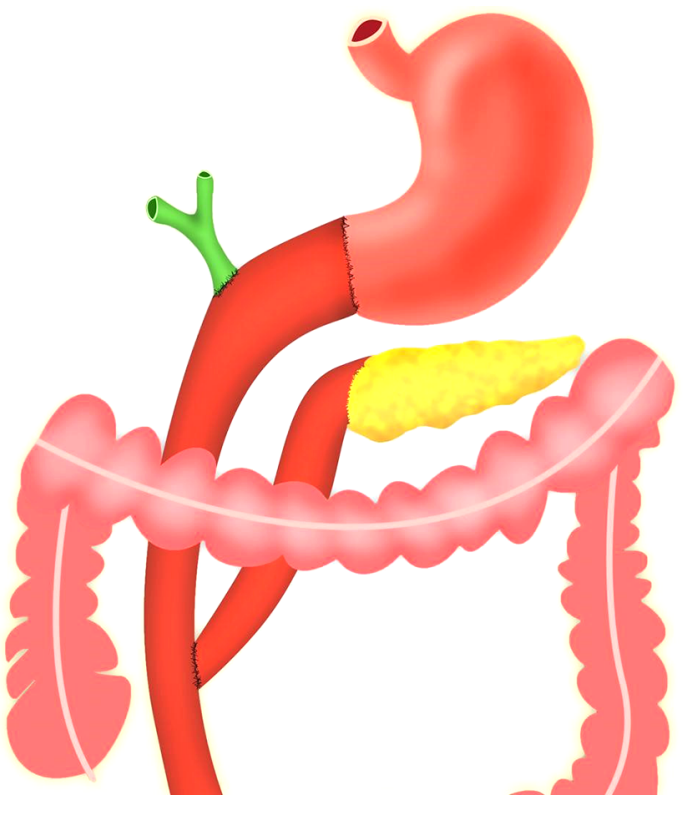

Figure 2. Double Roux reconstruction procedure (DRRP)

to end anastomosis we used the telescopic technique. The anastomosis was done in two layers. Then, distal part of this arm was cut off and the second arm of the Roux was raised up in retro-colic space and gastrojejunostomy was done in the end-to-end matter. After that, the end to side hepaticojejunostomy anastomosis was done $10 \mathrm{~cm}$ distal to the gastrojejunostomy anastomosis. The length of the second arm (gastric and biliary arm) was considered 45 to $65 \mathrm{~cm}$. In addition, the first Roux arm (pancreatic arm) was anastomosed end to side to the second arm. We used two drains for drainage: one near to the pancreaticojejunostomy anastomosis and another one near to the hepaticojejunostomy anastomosis.

\subsection{Patients Follow Up}

Biliary and pancreatic secretions were measured daily and recorded. Pancreatic fistula was defined as drainage of any assessable volume of fluid after third postoperative day with an amylase content higher than 3 times to the serum amylase (16). In addition, biliary leakage was defined as the presence of biliary secretions more than $50 \mathrm{~mL}$ in the drainage secretions after 24 hours of operation (17).

The drains were kept in place until secretions diminished below $10 \mathrm{~mL}$ per day for two days. The presence of any bleeding in the drains was also studied. In addition, in the presence of persistent fever, a CT scan with intravenous contrast was done to examine the presence of abscess or any liquid collection. 


\subsection{Statistical Analysis}

All of the data were entered to SPSS software version 22 and the results of qualitative variables were reported as the percentage and the results of quantitative variables were reported as mean $\pm S D$.

\section{Results}

A total of 12 patients underwent Whipple surgery with double Roux reconstruction procedure (DRRP) and followed up. Seven patients were male (58\%) and 5 patients were female (42\%). The mean age of patients was $53.08 \pm$ 13.43 with a range of 19 to 70 -year-old. In terms of the surgical indication, 6 patients (50\%) had periampullary cancer, 4 patients (33.4\%) had cancer in the head of the pancreas, 1 patient ( $8.3 \%$ ) had distal cholangiocarcinoma, and in 1 patient (8.3\%) traumatic duodenal injury. The mortality rate was 0 ; in addition, there were not any complication in terms of pancreatic fistula, intra-abdominal hemorrhage or intra-abdominal abscess. There was only 1 case (8.3\%) of biliary drainage from abdominal drains, which was closed expectantly without any additional surgical procedure.

\section{Discussion}

During this study, we studied outcomes of pancreaticoduodenectomy with double Roux reconstruction procedure (DRRP). The results showed lower mortality and morbidity in comparison to other reconstruction methods.

There are several ways to prevent complications of Whipple procedure such as pancreaticogastrostomy and pancreatic stent $(18,19)$. One of the ways to prevent complications of Whipple procedure is the separation of biliary and pancreatic anastomosis by two isolated (Roux) arms $(20,21)$. In this method, surgeon uses 2 distinct Roux limbs, one for the pancreatic secretions and the other one for the biliary and gastric anastomosis. Therefore, the main cause of complications which is the activation of pancreatic enzymes would be prevented. In addition, this reconstruction would prevent the accumulation of biliary and pancreatic secretions and gastric output in the single limb of jejunum. Therefore, that may prevent an increase in intraluminal pressure in Jejunal limb, which may predispose anastomotic leakage in these patients.

The used method in this study has several advantages such as a decrease in the stasis of pancreatic secretions and prevention of activation of pancreatic enzymes so that anastomotic leakage in DRRP would be milder and easy to manage than conventional Whipple procedure $(14,15)$. Another useful advantage was the feasibility of upper gastrointestinal endoscopy and endoscopic interventions in these patients during the post-operative period. Especially, for biliary anastomosis.

Limongelli et al. (22) used double Roux loop reconstruction after pyloric preserving pancreaticoduodenectomy (PPPD) and their results were promising with a lower incidence of pancreatic fistula and delayed gastric emptying. The results of this study were in accordance with Limongelli's study. We did not have any report regarding delayed gastric emptying because during our study the procedure was classic Whipple with resection of the distal stomach, not PPPD (22).

In another study which was conducted by Grobmyer et al. (23) there was not any difference between the conventional loop reconstruction and single or double Roux loop reconstruction in terms of mortality; In addition, the rate of pancreatic fistula was similar between both groups (23). Ke et al. (24) reported the same results and suggested that RYR does not reduce the rate of morbidities such as pancreatic fistula and only effects on the severity of morbidities and hospitalization These results were in line with the results of Uzunoglo et al. and Tani et al.'s study $(13,18)$; however, they were not related to the present study.

The present study contained some limitations such as small number of patients. Although, to the best of our knowledge, this is the first study in Iran which examines the complications and results of DRRP. It will be better to compare the single loop and DRRP in forms of randomized clinical trials with a large number of cases in the future studies.

\subsection{Conclusions}

DRRP in Whipple procedure can be a reliable method, especially in Iran, where the morbidity and mortality rate of following Whipple procedure is still high. However, more studies should be carried out to prove the efficacy of this method.

\section{Acknowledgments}

The authors of the present study would like to thank the operating room staff who helped us in the execution of this study.

\section{Footnotes}

Authors' Contribution: Study conception and design, drafting of manuscript, acquisition of data, and critical revision: Aboulfazl Afsharfard, Barmak Gholizadeh, and Adel Zeinalpour; acquisition of data, revision of manuscript: Mohammad Amin Shahrbaf and Behzad Azimi.

Conflict of Interests: Authors have no conflict of interest. 
Ethical Approval: All procedures performed in this study were in accordance with the ethical standards of the Institutional and National Research Committee and with the 1964 Helsinki declaration and its later amendments or comparable ethical standards. The current study was done after getting permission from Medical Ethics Committee of Shahid Beheshti University of Medical Sciences (Registration no: IR.SBMU.MSP.REC.1396.224).

Financial Disclosure: It is not declared by the authors.

Funding/Support: This research did not receive any specific grant from funding agencies in public, commercial, or not for-profit sectors.

\section{References}

1. Kausch W. [Das carcinom der papilla duodeni und seine radikale entfernung]. Beitr Klin Chir. 1912;78:439-86. German.

2. Schnelldorfer T, Sarr MG. Alessandro Codivilla and the first pancreatoduodenectomy. Arch Surg. 2009;144(12):1179-84. doi: 10.1001/archsurg.2009.219. [PubMed: 20026839].

3. Pedziwiatr M, Malczak P, Mizera M, Witowski J, Torbicz G, Major P, et al. Pancreatoduodenectomy for pancreatic head tumors in the elderly - Systematic review and meta-analysis. Surg Oncol. 2018;27(3):346-64. doi:10.1016/j.suronc.2018.05.021. [PubMed:30217288].

4. Giuliano K, Ejaz A, He J. Technical aspects of pancreaticoduodenectomy and their outcomes. Chin Clin Oncol. 2017;6(6):64. doi: 10.21037/cco.2017.09.01. [PubMed: 29156887].

5. Kim CB, Ahmed S, Hsueh EC. Current surgical management of pancreatic cancer. J Gastrointest Oncol. 2011;2(3):126-35.

6. Hartwig W, Werner J, Jager D, Debus J, Buchler MW. Improvement of surgical results for pancreatic cancer. Lancet Oncol. 2013;14(11):e47685. doi: 10.1016/S1470-2045(13)70172-4. [PubMed: 24079875].

7. Harnoss JC, Ulrich AB, Harnoss JM, Diener MK, Buchler MW, Welsch T. Use and results of consensus definitions in pancreatic surgery: A systematic review. Surgery. 2014;155(1):47-57. doi: 10.1016/j.surg.2013.05.035. [PubMed: 24694359].

8. Crist DW, Sitzmann JV, Cameron JL. Improved hospital morbidity, mortality, and survival after the Whipple procedure. Ann Surg. 1987;206(3):358-65. doi: 10.1097/00000658-198709000-00014. [PubMed: 3632096]. [PubMed Central: PMC1493175].

9. Klaiber U, Probst P, Knebel P, Contin P, Diener MK, Buchler MW, et al. Meta-analysis of complication rates for single-loop versus dualloop (Roux-en-Y) with isolated pancreaticojejunostomy reconstruction after pancreaticoduodenectomy. Br J Surg. 2015;102(4):331-40. doi: 10.1002/bjs.9703. [PubMed: 25644428].

10. Nahm CB, Connor SJ, Samra JS, Mittal A. Postoperative pancreatic fistula: a review of traditional and emerging concepts. Clin Exp Gastroenterol. 2018;11:105-18. doi: 10.2147/CEG.S120217. [PubMed: 29588609]. [PubMed Central: PMC5858541].

11. Hackert T, Werner J, Buchler MW. Postoperative pancreatic fistula. Surgeon. 2011;9(4):211-7. doi: 10.1016/j.surge.2010.10.011. [PubMed: 21672661]
12. Pratt WB, Maithel SK, Vanounou T, Huang ZS, Callery MP, Vollmer $\mathrm{CM}$ Jr. Clinical and economic validation of the International Study Group of Pancreatic Fistula (ISGPF) classification scheme. Ann Surg. 2007;245(3):443-51. doi: 10.1097/01.sla.0000251708.70219.d2. [PubMed: 17435552]. [PubMed Central: PMC1877022].

13. Uzunoglu FG, Reeh M, Wollstein R, Melling N, Perez D, Vashist YK, et al. Single versus double Roux-en-Y reconstruction techniques in pancreaticoduodenectomy: A comparative single-center study. World J Surg. 2014;38(12):3228-34. doi: 10.1007/s00268-014-2742-5. [PubMed: 25189443].

14. Kingsnorth AN. Safety and function of isolated Roux loop pancreaticojejunostomy after Whipple's pancreaticoduodenectomy. Ann $R$ Coll Surg Engl. 1994;76(3):175-9. [PubMed: 7912489]. [PubMed Central: PMC2502310].

15. Albertson DA. Pancreaticoduodenectomy with reconstruction by Roux-en-Y pancreaticojejunostomy: No operative mortality in a series of 25 cases. South Med J.1994;87(2):197-201. [PubMed: 8115883].

16. Bassi C, Dervenis C, Butturini G, Fingerhut A, Yeo C, Izbicki J, et al. Postoperative pancreatic fistula: An international study group (ISGPF) definition. Surgery. 2005;138(1):8-13. doi: 10.1016/j.surg.2005.05.001. [PubMed: 16003309].

17. de Castro SM, Kuhlmann KF, Busch OR, van Delden OM, Lameris JS, van Gulik TM, et al. Incidence and management of biliary leakage after hepaticojejunostomy.J Gastrointest Surg. 2005;9(8):1163-71. discussion 1171-3. doi: 10.1016/j.gassur.2005.08.010. [PubMed: 16269388].

18. Tani M, Kawai M, Hirono S, Okada KI, Miyazawa M, Shimizu A et al. Randomized clinical trial of isolated Roux-en-Y versus conventional reconstruction after pancreaticoduodenectomy. Br J Surg. 2014;101(9):1084-91. doi:10.1002/bjs.9544. [PubMed: 24975853].

19. Yeo CJ, Cameron JL, Lillemoe KD, Sauter PK, Coleman J, Sohn TA, et al. Does prophylactic octreotide decrease the rates of pancreatic fistula and other complications after pancreaticoduodenectomy? Results of a prospective randomized placebo-controlled trial. Ann Surg. 2000;232(3):419-29. doi: 10.1097/00000658-200009000-00014. [PubMed: 10973392]. [PubMed Central: PMC1421155].

20. Nakamura T, Okada A, Higaki J, Tojo H, Okamoto M. Pancreaticobiliary maljunction-associated pancreatitis: An experimental study on the activation of pancreatic phospholipase A2. World J Surg. 1996;20(5):543-50. [PubMed: 8661628].

21. Pescio G, Cariati E. A new reconstructive method after pancreaticoduodenectomy: The triple Roux on a "P" loop. Rationale and radionuclide scanning evaluation. HPB Surg. 1996;9(4):2237. doi: 10.1155/1996/53812. [PubMed: 8809583]. [PubMed Central: PMC2443773].

22. Limongelli P, D’Alessandro A, Parisi S, Pirozzi R, Bondanese M, Colella $\mathrm{C}$, et al. Double loop reconstruction following pancreaticoduodenectomy for malignant tumor: Short-term outcome. Int J Surg Case Rep. 2016;20S:16-20. doi: 10.1016/j.ijscr.2016.02.002. [PubMed: 26872635] [PubMed Central: PMC4883052].

23. Grobmyer SR, Hollenbeck ST, Jaques DP, Jarnagin WR, DeMatteo R, Coit DG, et al. Roux-en-Y reconstruction after pancreaticoduodenectomy. Arch Surg. 2008;143(12):1184-8. doi: 10.1001/archsurg.2008.501. [PubMed: 19075170].

24. Ke S, Ding XM, Gao J, Zhao AM, Deng GY, Ma RL, et al. A prospective, randomized trial of Roux-en-Y reconstruction with isolated pancreatic drainage versus conventional loop reconstruction after pancreaticoduodenectomy. Surgery. 2013;153(6):743-52. doi 10.1016/j.surg.2013.02.008. [PubMed: 23601899]. 